Letter to Editor:

\title{
Auditory Training Among Older Adults With Alzheimer Disease and Central Auditory Processing Disorder
}

\author{
Fereshteh Bagheri $^{1^{*}}$ (D), Mohammad Rezaei ${ }^{2 *}$ (D), Vahid Rashedi ${ }^{3,4^{*}}$ (D) \\ 1. Department of Audiology, School of Rehabilitation Sciences, Babol University of Medical Sciences, Mazandaran, Iran \\ 2. Autism Spectrum Disorders Research Center, Hamadan University of Medical Sciences, Hamadan, Iran. \\ 3. School of Behavioral Sciences and Mental Health (Tehran Institute of Psychiatry), Iran University of Medical Sciences, Tehran, Iran \\ 4. Iranian Research Centre on Aging, University of Social Welfare and Rehabilitation Sciences, Tehran, Iran.
}

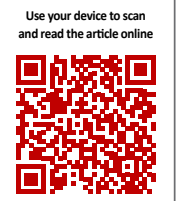

Citation Bagheri F, Rezaei M, Rashedi V. Auditory Training Among Older Adults With Alzheimer Disease and Central Auditory Processing Disorder. Avicenna J of Neuropsychophysiology. 2018; 5(4):147-150. http://dx.doi.org/10.32598/ajnpp.4.3.270

doi $h$ http://dx.doi.org/10.32598/ajnpp.4.3.270

\section{Letter to Editor}

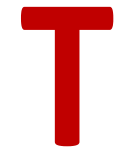

he syndrome of dementia has many types, and Alzheimer Disease (AD) is the most common type of dementia. AD is a progressive disorder of the central nervous system that involves different regions of the cortex, changes the morphology of cortical cells, and affect various neurotransmitters, particularly acetylcholine. Because of the involvement of the frontal cortex in an early stage of $A D$, deficits in working memory become manifest soon. The decline in memory processes in individuals with $A D$ is well documented and one of the features of deterioration of memory functions is the loss of working memory [1]. Understanding the nature of working memory can probably lead us toward a proper treatment protocol. By definition, working memory is the maintenance of information in a short span that is essential for communication as information is held in consciousness [2].

Several factors influence the degree and quality of working memory decrease in AD. The psychotic status that is often associated with AD such as depression may lead to an even greater decline in working memory. The capacity of working memory could be decreased in neurodegenerative diseases. The decrease in capacity of working memory is attributed to a reduction in the activity of the dorsolateral prefrontal cortex with aging [3]

As $A D$ extends to the auditory cortex and higher-level auditory centers, the hearing problem caused by central auditory processing disorder appears, in which the peripheral auditory system remains normal [4]. Auditory processing refers to the set of auditory abilities such as localization, sounds discrimination, auditory perception, temporal processing, and dichotic performance with the competing messages. Individuals with central auditory processing disorder have problems in establishing the relationship between sounds and meanings, especially in a noisy environment because in auditory processing, there is a direct connection between auditory discrimination, memory, and interpretation of spoken word [5].

Cognitive deficits affect language abilities, and a significant number of individuals with $A D$ have language problems even in the early stage of the disease [6] It has been shown that auditory training can induce changes at the cortical level.

\section{* Corresponding Author:}




\section{Auditiry training > Semantic memory $>$ Working memory}

Figure. 1. Connection of events *

* This is a theory based on changes in brain activity after auditory training.

Both human and animal studies have demonstrated cortical plasticity that improved auditory perception. There is an interaction between cortical and subcortical plasticity and auditory training showed changes in the brainstem in response to speech sounds [7]. Working memory capacity can be remediated by adaptive training, too [2]. Auditory training is the targeted programs designed to increase the amount of information that human uses for total perception [8]. The effects of auditory training on working memory capacity have rarely been studied in individuals with AD without hearing problems.

Auditory training encourages individuals to establish the relation between sounds and meanings that increases cortical activity and activates brain plasticity mechanisms [9]. Despite its benefit, auditory training is rarely recommended for older adults with problems in perception. In this situation, designing rehabilitation programs with the aim of speech perception in individuals with $A D$ could increase their working memory capacity by improving semantic memory (Figure 1).

Alzheimer disease affects several auditory nuclei in the brainstem. One of these nuclei is the medial geniculate body that is an essential auditory station for central auditory function and relaying information to the cortex [10]. Central auditory processing disorder in the temporal lobe may start earlier than $A D$ in older people and leads to deficits in speech production [10]. Because of the higher risk of $A D$ in older adults with hearing problems, it is crucial to start auditory training for older people before the onset of AD. In conclusion, it is urgent to research auditory training and its influence on individuals with $A D$ and central auditory disorder.

\section{Ethical Considerations}

\section{Compliance with ethical guidelines}

All ethical principles were considered in this article. This article does not contain any studies involving human participants performed by any of the authors.

\section{Funding}

This research did not receive any specific grant from funding agencies in the public, commercial, or not-forprofit sectors.

\section{Authors' contributions}

All authors contributed in preparing this article.

\section{Conflict of interest}

The authors declared t no conflict of interest.

\section{References}

[1] Bayles KA. Effects of working memory deficits on the communicative functioning of Alzheimer's dementia patients. Journal of Communication Disorders. 2003; 36(3):209-19. [DOI:10.1016/S00219924(03)00020-0]

[2] Klingberg T. Training and plasticity of working memory. Trends in Cognitive Sciences. 2010; 14(7):317-24. [DOI:10.1016/j. tics.2010.05.002] [PMID]

[3] Gilmour G, Porcelli S, Bertaina-Anglade V, Arce E, Dukart J, Hayen A, et al. Relating constructs of attention and working memory to social withdrawal in Alzheimer's disease and schizophrenia: Issues regarding paradigm selection. Neuroscience \& Biobehavioral Reviews. 2018; 97:47-69. [DOI:10.1016/j.neubiorev.2018.09.025] [PMID]

[4] Bagheri F, Borhaninejad V, Rashedi V. Alzheimer's disease and Hearing Loss among older adults: A literature review. International Journal of Psychology and Behavioral Sciences. 2018; 8(5):77-80.

[5] Stroiek S, Quevedo LdS, Kieling CH, Battezini ACL. Auditory training in auditory processing disorders: A case study. Revista College of Education Faculty Affairs Committee. 2015; 17(2):604-14. [DOI:10.1590/1982-021620157914]

[6] Szatloczki G, Hoffmann I, Vincze V, Kalman J, Pakaski M. Speaking in Alzheimer's disease, is that an early sign? Importance of changes in language abilities in Alzheimer's disease. Frontiers in Aging Neuroscience. 2015; 7:195. [DOI:10.3389/fnagi.2015.00195] [PMID] [PMCID]

[7] Russo NM, Nicol TG, Zecker SG, Hayes EA, Kraus N. Auditory training improves neural timing in the human brainstem. Behavioural Brain Research. 2005; 156(1):95-103. [DOI:10.1016/j.bbr.2004.05.012] [PMID] 
[8] Nkyekyer J, Meyer D, Pipingas A, Reed NS. The cognitive and psychosocial effects of auditory training and hearing aids in adults with hearing loss. Clinical Interventions in Aging. 2019; 14:123-5. [DOI:10.2147/CIA.S183905] [PMID] [PMCID]

[9] Kraus N, White-Schwoch T. Training older adults to hear better. The Hearing Journal. 2019; 72(1):46-7. [DOI:10.1097/01. HJ.0000557750.43399.3a]

[10] Iliadou V, Kaprinis S. Clinical psychoacoustics in Alzheimer's disease central auditory processing disorders and speech deterioration. Annals of General Hospital Psychiatry. 2003; 2(1):12 [DOI:10.1186/1475-2832-2-12] 
